Tohoku J. Exp. Med., 2008, 214, 269-280

\title{
Normal Bone Growth Requires Optimal Estrogen Levels: Negative Effects of Both High and Low Dose Estrogen on the Number of Growth Plate Chondrocytes
}

\author{
Hiroyuki Takano, ${ }^{1}$ Toshimi Aizawa,,${ }^{1}$ Taichi Irie, ${ }^{1}$ EijI Itoi,,${ }^{1}$ Shoichi Kokubun ${ }^{1}$ \\ and HelmTRud I. ROACH ${ }^{2}$ \\ ${ }^{1}$ Department of Orthopaedic Surgery, Tohoku University School of Medicine, Sendai, Japan \\ ${ }^{2}$ Bone \& Joint Research Group, General Hospital, Southampton, U.K.
}

\begin{abstract}
Endochondral bone formation at epiphyseal growth plate consists of the synchronized processes of chondrogenesis and cartilage ossification. Estrogen, the major female sex hormone, plays an important role in this process, particularly during the pubertal growth spurt. However, its effects on the growth plate are not completely understood. The aims of this study were to clarify the effects of estrogen on the kinetics of chondrocytes in the growth plates of 10- to 25-week-old female rabbits by studying the effects of ovariectomy or highdose administration of estrogen on the balance between cell proliferation and death. Fortyeight Japanese white rabbits were divided into three groups: sham operated, ovariectomized, or ovariectomized with subsequent weekly injection of high dose estrogen from 10 weeks. The chondrocyte kinetics was investigated by histomorphometry and immunohistochemistry, using antibodies for caspase-3, a marker of apoptosis, and for proliferating cell nuclear antigen. Both ovariectomized and estrogen-injected rabbits showed a declination of the chondrocyte number although the latter animals indicated a more dramatic effect. Estrogen-injected rabbits showed a decrease in the cell proliferating ability together with an increase in chondrocytes undergoing apoptosis while ovariectomy mainly reduced the cell proliferating ability. Given the known importance of estrogen for bone growth, one would expect that ovariectomy and high-dose administration of estrogen would have opposite effects. However, the present study indicated that both low and high concentration had a similar effect: a decrease in the chondrocyte number compared with control, suggesting that estrogen has to be maintained within a narrow range for optimal bone growth. - growth plate; chondrocyte; estrogen; ovariectomy; apoptosis.
\end{abstract}

Tohoku J. Exp. Med., 2008, 214 (3), 269-280.

(C) 2008 Tohoku University Medical Press

Longitudinal bone growth of the long bones occurs at the epiphyseal growth plate by endochondral ossification. Resting chondrocytes at the top of the plate proliferate, secrete cartilage matrix, differentiate to hypertrophic chondrocytes and are finally removed by programmed cell death (apoptosis) (Hunziker et al. 1987; Grumbach 2000; Robertson 1990; van der Eerden et al. 2003). Simultaneously, the empty lacunae at the metaphyseal border of the growth plate are invad-

Received September 26, 2007; revision accepted for publication January 24, 2008.

Correspondence: Toshimi Aizawa, M.D., Ph.D., Department of Orthopaedic Surgery, Tohoku University

School of Medicine, 1-1, Seiryo-machi, Aoba-ku, Sendai 980-8574, Japan.

e-mail: toshi-7@ra2.so-net.ne.jp 
ed by blood vessels. Osteoblast progenitor cells migrate to non-resorbed struts of calcified cartilage and deposit new bone into bone to form the primary spongiosa. These synchronized processes of chondrogenesis and cartilage ossification lead to longitudinal bone growth (Grumbach 2000; Weise et al. 2001).

Many hormones, cytokines, and growth factors are associated with the regulation of the endochondral bone formation processes (van der Eerden et al. 2003). Estrogen is one of the major sex hormones and is mainly secreted by the ovaries in females, and in males it is derived by the aromatization of testosterone and androstenedione (Grumbach 2000). It is the critical hormone controlling the pubertal growth spurt in females and the growth plate fusion in both sexes (Grumbach 2000; Rodd et al. 2004). In addition, estrogen is important for the regulation of bone metabolism and maintaining the trabecular bone mineral density (Lindberg et al. 2001).

We have previously studied the cell kinetics of growth plate chondrocytes, defined by the balance of cell proliferation and cell death (Aizawa et al. 1997, 1998, 1999; Irie et al. 2005). To study the effects of estrogen, many researchers have used ovariectomy, ovariectomy with subsequent estrogen injection, or transgenic mice of estrogen receptors (Silberberg and Silberberg 1971; Weise et al. 2001; van der Eerden et al. 2003; Bardin et al. 2004; Eastell 2005; Oestergaard et al. 2006). Ovariectomy led to accelerate the growth and delayed growth plate fusion, whereas high dose of estrogen administration suppressed growth with early growth plate fusion (Silberberg and Silberberg 1971; Nilsson et al. 2002; Chagin et al. 2004; Nilsson and Baron 2005). More recent studies suggested that estrogen might cause earlier exhaustion of the proliferating ability of chondrocytes (Weise et al. 2001; Rodd et al. 2004). However, the effects of estrogen on the function of growth plate chondrocytes and ultimately on the process of endochondral bone formation are still poorly understood (Rodd et al. 2004). In particular, there have been no reports, to our knowledge, on the effects of low or high dose of estrogen on the cell kinetics of chondrocytes.
Therefore, the aims of this study were to clarify the action of this female hormone on the chondrocyte kinetics using ovariectomized and ovariectomized with subsequent high dose estrogen-injected female rabbits.

\section{Materials and Methods}

\section{Animals}

Rabbits were selected for this study because in rabbits, like humans but unlike other rodents, the epiphyses are fused at the time of sexual maturation and in response to sex steroids (Weise et al. 2001). The experiment was carried out according to the guidelines of the Ethics Committee of Tohoku University. Forty-eight female Japanese white rabbits were divided into twelve groups of four. Sixteen rabbits were sham-operated; only skin incision and subsequent suturing were performed at 8 weeks of age. They were euthanized by intravenous injection of an overdose of pentobarbital sodium at 10 , 15,20 , and 25 weeks and were designated as the $10 \mathrm{~W}$, $15 \mathrm{~W}, 20 \mathrm{~W}$, and $25 \mathrm{~W}$ groups, respectively.

Sixteen rabbits underwent ovariectomy at 8 weeks and were euthanized at 10, 15, 20, and 25 weeks of age. They were designated as the OV-10W, OV-15W, OV$20 \mathrm{~W}$, and OV-25W groups, respectively. Another sixteen rabbits were ovariectomized at 8 weeks, then 17 betaestradiol $\left(\mathrm{E}_{2}\right)(70 \mu \mathrm{g} / \mathrm{kg})$ was given by weekly intramuscular injections from week 10 onwards according to the protocol of Weise et al. (2001) and Nilsson et al. (2003). $\mathrm{E}_{2}$ is the major component of estrogen, and 10 weeks was assumed to be before sexual maturation so that the rabbits were not exposed to abundant endogenous $\mathrm{E}_{2}$ (Suzuki et al. 1989). The rabbits were also euthanized at 10, 15, 20 , and 25 weeks and were designated as the $\mathrm{E}_{2}-10 \mathrm{~W}, \mathrm{E}_{2}-$ $15 \mathrm{~W}, \mathrm{E}_{2}-20 \mathrm{~W}$, and $\mathrm{E}_{2}-25 \mathrm{~W}$ groups, respectively.

The level of serum $E_{2}$ was measured at the time of gonadectomy and when the rabbits were euthanized. Blood was collected from an ear vein of all rabbits. The serum $\mathrm{E}_{2}$ concentrations were determined by radioimmunoassay (Korenman et al. 1970). The right leg of each rabbit was dismembered and the length of the femur was measured using soft radiography (low power $<30 \mathrm{kV}$ ).

\section{Tissue preparation and morphological observation}

The epiphyseal growth plate was taken from the center of the femoral head of each rabbit as in our previous studies (Aizawa et al. 1997, 1998, 1999; Irie et al. 2005). The growth plates were sectioned coronally into blocks $5 \mathrm{~mm}$ thick. These were fixed in $10 \%$ formalin 
for 3 days, decalcified by ethylenediaminetetraacetic acid (EDTA) at $4^{\circ} \mathrm{C}$ for 10 days, embedded in paraffin and cut into sections $2.5 \mu \mathrm{m}$ thick.

After deparaffinization and rehydration, some sections from each specimen were first stained with haematoxylin and eosin (HE). These were used to investigate the morphological changes in growth plate chondrocytes with age and to measure the height of the growth plate. Immunostaining for osteonectin (ONT), a typical noncollagenous bone protein, was performed in order to localize hypertrophic chondrocytes since only they secrete ONT (Pacifici et al. 1990; Aizawa et al. 1998). The measurements of the growth plate height and immunostaining for ONT were performed as in our previous study (Irie et al. 2005). Using both HE and ONT immunostained sections, each growth plate was divided into three zones: resting, proliferating, and hypertrophic. The cell numbers were counted three times in five fields randomly selected at a magnification of $\times 200$ on the HE sections in the whole growth plate and the three divided zones (Irie et al. 2005).

\section{Immunohistochemistry}

Apoptotic chondrocytes were visualized in situ by immunostaining for caspase-3 (Biotech Inc., Santa Cruz, CA, USA), while cell proliferation was detected by immunostaining for proliferating cell nuclear antigen (PCNA, Dako, Cytomation, Glostrup, Denmark), as in our previous study (Irie et al. 2005). Briefly, the avidinbiotinylated peroxidase complex (ABC) method was used for the immunostaining for caspase-3 and the labeled streptavidin-biotin (LSAB) method for PCNA. Negative controls were incubated with mouse serum and showed only background staining. Sections were counterstained with hematoxylin for caspase-3 and methyl green for PCNA. For each antibody and each rabbit block, immunocytochemistry for caspase-3, PCNA, and ONT was performed on several sections per rabbit. We accepted the results as final when at least two sections showed the same immunostaining pattern.

\section{Quantification of caspase-3 and PCNA expressions}

Chondrocytes with diffusely stained cytoplasm or nuclei were regarded as caspase- 3 positive cells, whereas only nuclear staining was considered positive for PCNA. The caspase-3-positive and PCNA-positive ratios were calculated in the whole growth plate and in the three divided zones (Aizawa et al. 1997, 1998, 1999; Irie et al. 2005). The ratios of the ovariectomized and $E_{2}$-injected rabbits were compared with those of the normal ones at similar ages.

\section{Statistical analysis}

We used a one-way ANOVA between the normal, ovariectomized, and $\mathrm{E}_{2}$-injected rabbits at similar ages in order to determine the significance of the differences in the caspase-3-positive and PCNA-positive ratios. We also used a Post hoc for statistical evaluation of the length of the femur and the height of the growth plate in each rabbit group. A possible value less than 0.05 was considered as significant. All statistical analyses were performed with commercially available software (Statview, Abacus Concepts, Berkeley, CA, USA).

\section{Results}

\section{Serum $E_{2}$ levels}

In the normal rabbits, the serum $\mathrm{E}_{2}$ gradually increased with age to $19 \mathrm{pg} / \mathrm{ml}$ at 25 weeks of age in the study period. In the ovariectomized rabbits, serum $\mathrm{E}_{2}$ levels were less than $8 \mathrm{pg} / \mathrm{ml}$ in every age group. In the $\mathrm{E}_{2}$-injected group, one rabbit showed $24 \mathrm{pg} / \mathrm{ml}$ and the others more than $35 \mathrm{pg} /$ $\mathrm{ml}$ at 10 weeks of age. The $\mathrm{E}_{2}$ levels of the other age group were over $70 \mathrm{pg} / \mathrm{ml}$. As expected, at 15,20 , and 25 weeks, the average serum $\mathrm{E}_{2}$ levels of the ovariectomized rabbits were significantly lower than those of the normal ones, while those of the $E_{2}$-injected ones were significantly higher in every age group (Fig. 1).

\section{Length of femurs and height of growth plates}

The femurs of the ovariectomized rabbits tended to be longer than those of the normal rabbits at the same age, while those of the $\mathrm{E}_{2}$-injected ones tended to be shorter (Table 1). The heights of the whole growth plate and the three divided zones of the normal, ovariectomized, and $\mathrm{E}_{2^{-}}$ injected rabbits are shown in Fig. 2. Those of the whole plate decreased with age in every rabbit group. However, the heights of the growth plate of ovariectomized rabbits were greater, while that of $E_{2}$-injected rabbits were less compared with the corresponding normal ones. The most obvious decrease was in the proliferating zone. 


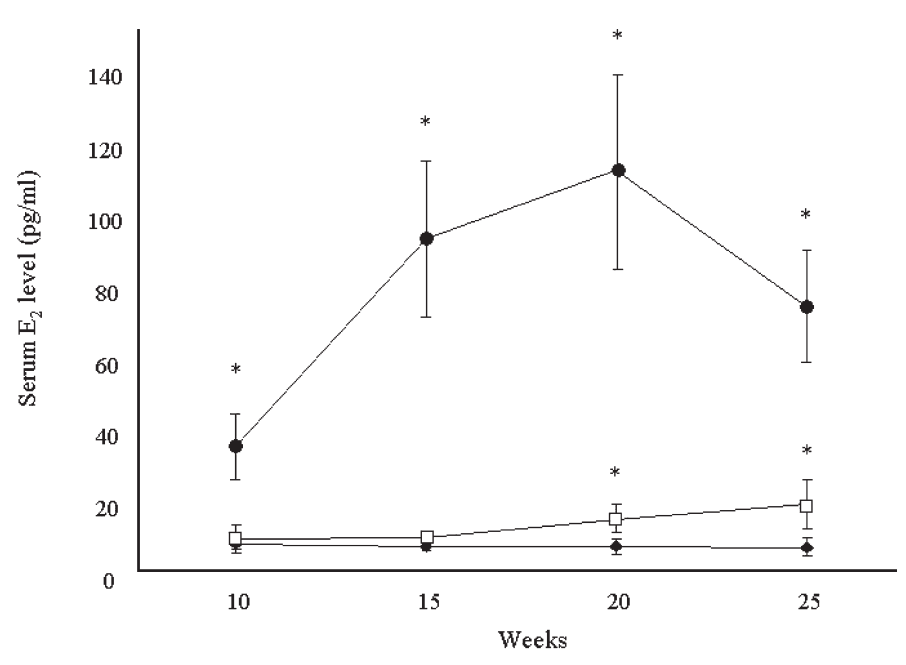

Fig. 1. The serum level of $E_{2}$ in the normal, ovariectomized, and $E_{2}$-injected rabbits. In the normal rabbits, serum $E_{2}$ increases with age. In the ovariectomized rabbits, serum $E_{2}$ is less than $8 \mathrm{pg} / \mathrm{ml}$ in each age group, while in the $\mathrm{E}_{2}$-injected groups, levels are more than $35 \mathrm{pg} / \mathrm{ml}$ in each age group. At 15,20 , and 25 weeks, the average serum $E_{2}$ levels of the ovariectomized rabbits are significantly lower than those of the normal ones, while those of the $\mathrm{E}_{2}$-injected group are significantly higher in every age group. Significant difference between each group is: $* p<0.05$.

$-\square-$, normal rabbits; $-\checkmark-$, ovariectomized rabbits; $-\mathbf{-}-, \mathrm{E}_{2}$-injected rabbits.

TABLE 1. The mean (s.D.; $\mathrm{cm}$ ) lengths of the femur according to age in three rabbit groups.

\begin{tabular}{lcccc}
\hline \multirow{2}{*}{ Group } & \multicolumn{5}{c}{ Age (weeks) } \\
\cline { 2 - 5 } & 10 & 15 & 20 & 25 \\
\hline Normal & $7.8(0.2)$ & $9.2(0.2)$ & $9.2(0.4)$ & $9.4(0.3)$ \\
Ovariectomized & $7.9(0.2)$ & $9.0(0.2)$ & $9.4(0.3)$ & $9.7(0.4)$ \\
$\mathrm{E}_{2}$-injected & $7.6(0.1)$ & $9.0(0.3)$ & $9.0(0.6)$ & $8.9(0.3)^{\S}$ \\
\hline
\end{tabular}

Statistical analysis applying the Post hoc.

${ }^{8} p<0.05$ compared with the ovariectomized rabbits.

\section{Histological findings}

In the normal rabbits, the obvious columnar structures were maintained until 20 weeks of age in the proliferating and upper hypertrophic zones. With increasing age, fewer chondrocytes were present, particularly in the proliferating zone; and the columnar structures became disarranged (Fig. 3A). Cells with large round to oval-shaped cytoplasm, which were positively stained by ONT, were present in the hypertrophic zone in each age group (Fig. 4A).

In the OV-10W group, the columnar structures and the morphology of the chondrocytes were almost the same as those of the $10 \mathrm{~W}$ group
(Fig. 3B-10W). In the OV-15W group, the flattened, proliferating cells decreased and the columnar structures from the proliferating to upper hypertrophic zones were somewhat disarranged (Fig. 3B-15W). In this group, slightly flattened chondrocytes, located in the upper part of the hypertrophic zone, were negative for ONT, indicating that they were not hypertrophic cells (Fig. 4B). In the OV-20W group, the columnar structures were irregular and the cellularity was reduced, particularly in the proliferating zone (Fig. $3 \mathrm{~B}-20 \mathrm{~W})$. In the OV-25W group, the cellularity was more reduced and some areas had only a few chondrocytes within large regions of extracellular 


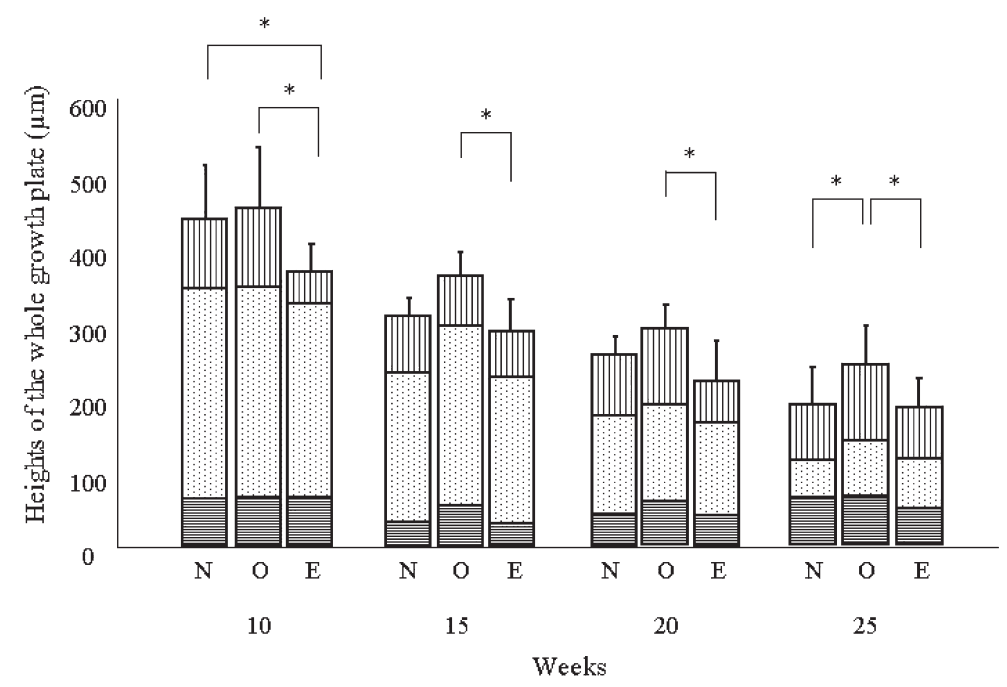

Fig. 2. The heights of the growth plates of each age group. Each bar represents the mean (S.D.). The bars inside the histograms divide the whole plates into three zones. The heights of the whole plates decrease with age in every rabbit group but those of the ovariectomized groups are higher in each age group while those of the $\mathrm{E}_{2}$-injected rabbits are lower compared to the normal ones. Statistically significant differences are found between the ovariectomized and $\mathrm{E}_{2}$-injected rabbits of every age group. Significant difference between each group is: ${ }^{*} p<0.05$. N, normal rabbits; O, ovariectomized rabbits; E, $\mathrm{E}_{2}$-injected rabbits.

血, resting zone; , proliferating zone; 琶, hypertrophic zone.

matrix (Fig. 3B-25W). The overall morphology, including the size of the hypertrophic chondrocytes, was similar to that in the normal rabbits.

In the $\mathrm{E}_{2}-10 \mathrm{~W}$ group, the columnar structures and the morphology of the chondrocytes were almost the same as those of the 10W group and OV-10W group (Fig. 3C-10W). With increasing age, the heights of the overall growth plate and number of chondrocytes had decreased more rapidly, particularly in the proliferating zone, than in the corresponding normal and ovariectomized groups (Fig. 3C). In the $\mathrm{E}_{2}-20 \mathrm{~W}$ group, the decrease of the cellularity was remarkable, particularly in the proliferating zone (Fig. 3C-20W). In the $\mathrm{E}_{2}-25 \mathrm{~W}$ group, only several hypertrophic chondrocytes were found in most areas (Fig. $3 \mathrm{C}-25 \mathrm{~W})$. As in the normal and ovariectomized groups, chondrocytes, which stained positively for ONT, were present in the hypertrophic zone in the $\mathrm{E}_{2}$-injected rabbits (Fig. 4C).

\section{Chondrocyte numbers}

The cell numbers of the whole growth plate and the three divided zones are shown in Fig. 5. The numbers of chondrocytes decreased in the whole growth plate and also in the three divided zones with age in all three rabbit groups. The chondrocytes in the normal group outnumbered those in the other two groups in the whole growth plate and the three divided zones, while the $\mathrm{E}_{2}-$ injected rabbits showed the fewest numbers among the three groups. In the whole growth plate, there were significant differences at every age among the three groups except between the $\mathrm{OV}-25 \mathrm{~W}$ and $\mathrm{E}_{2}-25 \mathrm{~W}$ groups. In the three divided zones, fewer chondrocytes were present in the proliferating and hypertrophic zones of the $\mathrm{E}_{2^{-}}$ injected rabbits compared with the corresponding zones of the normal and ovariectomized rabbits. Particularly, the numbers of proliferating cells of the $\mathrm{E}_{2}-10 \mathrm{~W}, \mathrm{E}_{2}-15 \mathrm{~W}$, and $\mathrm{E}_{2}-20 \mathrm{~W}$ groups and those of hypertrophic chondrocytes of the $E_{2}-10 \mathrm{~W}$ and $E_{2}-15 \mathrm{~W}$ groups were less than half those of the corresponding normal rabbits. 
A

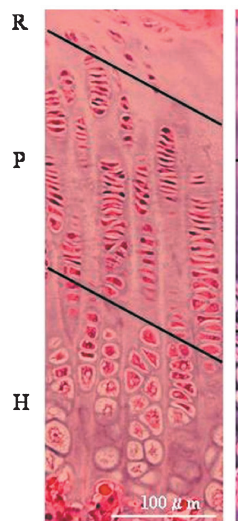

10W

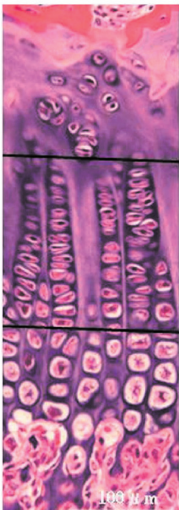

$15 \mathrm{~W}$

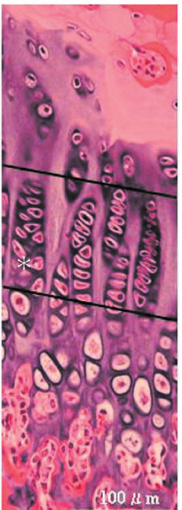

20W

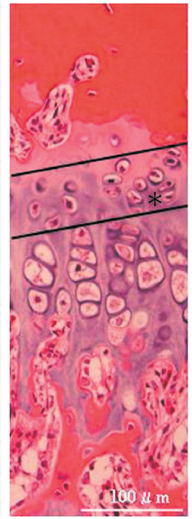

$25 \mathrm{~W}$
B

$\mathrm{R}$

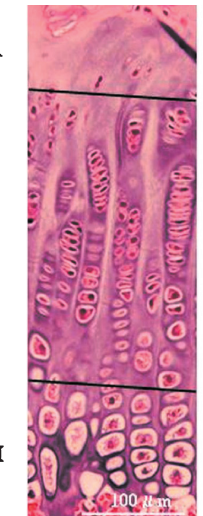

$10 \mathrm{~W}$

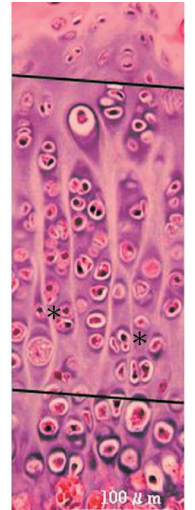

$15 \mathrm{~W}$

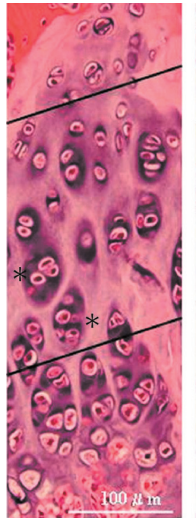

20W

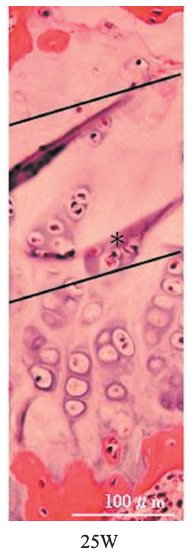

C

R
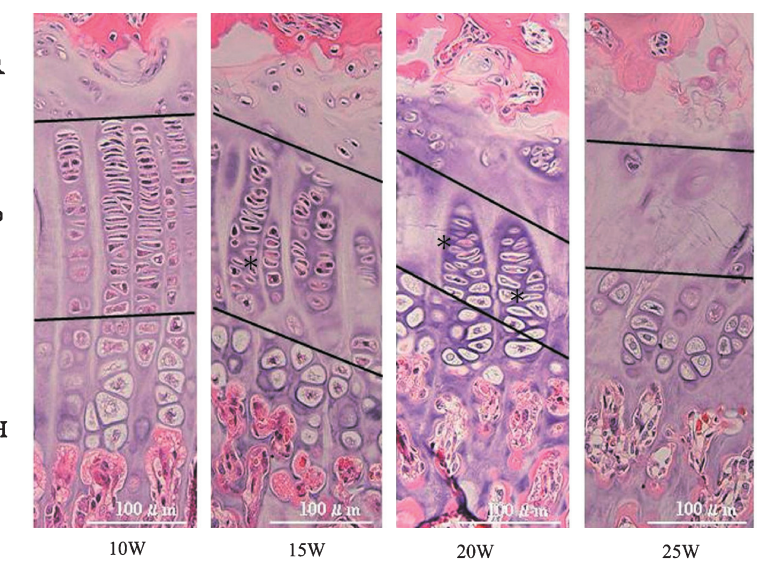

Fig. 3. The growth plate in the normal (A), ovariectomized (B), and $\mathrm{E}_{2}$-injected rabbits (C). Photomicrographs at 10 weeks (A, B, and C-10W), 15 weeks (A, B, and C-15W), 20 weeks (A, B, and $\mathrm{C}-20 \mathrm{~W}$ ), and 25 weeks (A, B, and C-25W) (haematoxylin and eosin [HE], original magnification $\times 200$ ). In the normal rabbits, chondrocytes numbers are reduced with increasing age, particularly in the proliferating zone, and the columnar structures in the proliferating to upper hypertrophic zones are disarranged at 20 and 25 weeks. In the ovariectomized rabbits, the columnar structure is still present in the 8-10W group but is disarranged from 15 weeks of age. The cellularity is reduced, particularly in the proliferating zone at 20 and 25 weeks. In the $\mathrm{E}_{2}$-injected rabbits at 10 weeks of age, the columnar structures and the morphology of the chondrocytes are almost the same as those of the $10 \mathrm{~W}$ group and OV-10W group. With increasing age, the heights of the overall growth plate and number of chondrocytes decrease more rapidly, particularly in the proliferating zone, compared with the normal corresponding age group.

Asterisks show the disarranged columnar structures in the proliferating to upper hypertrophic zones. $\mathrm{R}$, resting zone; $\mathrm{P}$, proliferating zone; $\mathrm{H}$, hypertrophic zone. 
A

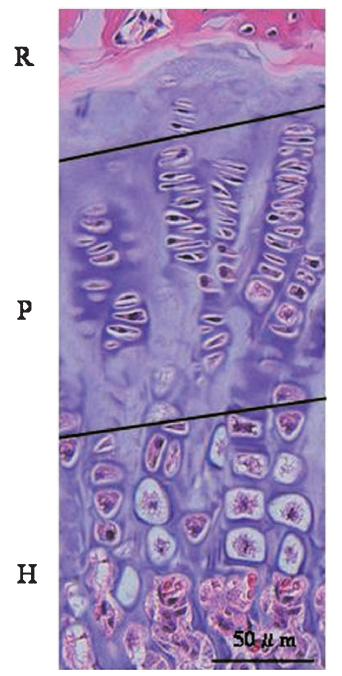

HE

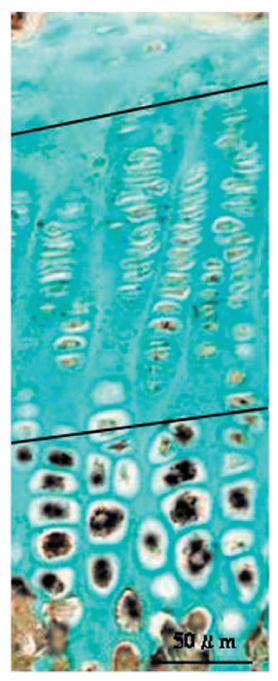

ONT
B

R

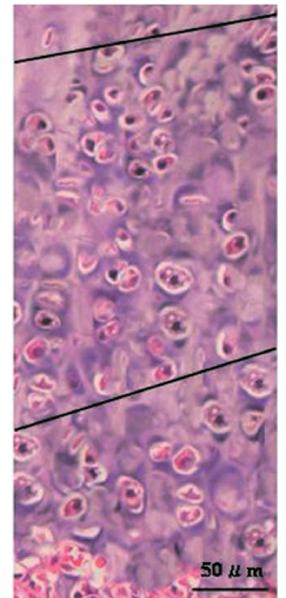

$\mathrm{HE}$

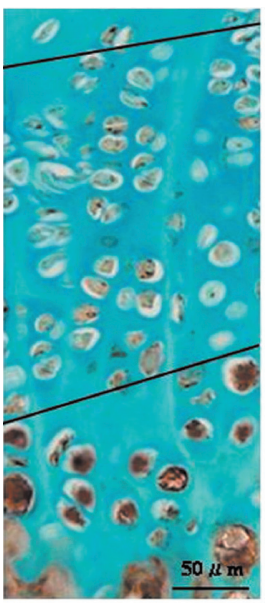

ONT

$\mathrm{C}$

$\mathbf{R}$

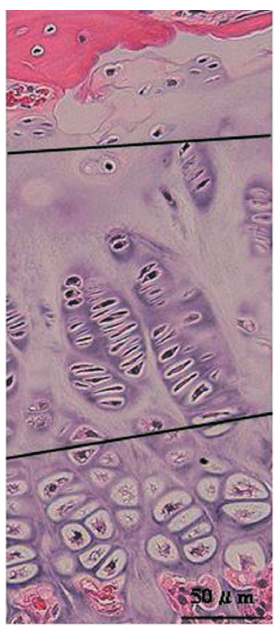

HE

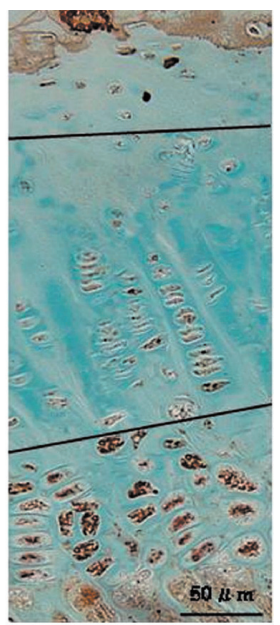

ONT

Fig. 4. Three divided zones of the growth plate in the normal (A), ovariectomized (B), and $\mathrm{E}_{2}$-injected rabbits $(\mathrm{C})$. Photomicrographs at 15 weeks using HE (A, B, and C-HE), immunostaining for osteonectin $(\mathrm{A}, \mathrm{B}$, and $\mathrm{C}-\mathrm{ONT}$ ) (original magnification $\times 200$ ). The hypertrophic zone is divided according to the detection of osteonectin-positive cells.

$\mathrm{R}$, resting zone; $\mathrm{P}$, proliferating zone; $\mathrm{H}$, hypertrophic zone. 


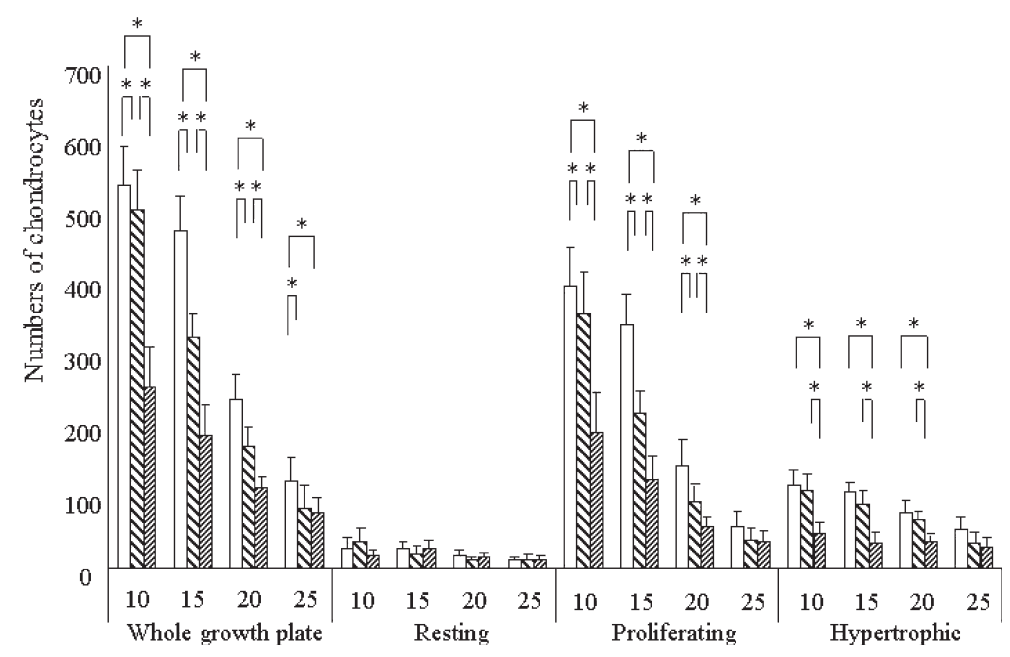

Fig. 5. Histogram showing the mean (s.D.) number of chondrocytes in the whole growth plate and the three divided zones in the normal, ovariectomized, and $\mathrm{E}_{2}$-injected rabbits. The numbers of chondrocytes decrease in the whole growth plate and also in the three divided zones with age in all three rabbit groups. The chondrocytes in the normal group outnumber those in the other two groups in the whole growth plate and the three divided zones, while the $\mathrm{E}_{2}$-injected rabbits show the fewest numbers among the three groups. Significant difference between each group is: ${ }^{*} p<0.05$.

$\square$, normal rabbits; $\mathbb{N}$, ovariectomized rabbits; $\mathbb{Z}, \mathrm{E}_{2}$-injected rabbits.

\section{Immunostaining for caspase-3 and PCNA}

Caspase-3-positive chondrocytes were found in every growth plate of each rabbit group and in all three divided zones. Most of the positive chondrocytes were found in the hypertrophic zone (Fig. 6). The caspase-3-positive ratio of the whole growth plate tended to increase with age in all three rabbit groups (Fig. 7). The $\mathrm{E}_{2}$-injected rabbits showed the highest positive ratios in every age group, which were significantly higher than those of the other two groups at the same age. The ratios in the ovariectomized rabbits were higher than those in the corresponding normal group, but a statistically significant difference was found only between the $20 \mathrm{~W}$ and $\mathrm{OV}-20 \mathrm{~W}$ groups. In the hypertrophic zone, more than half of the cells were positively stained in the $\mathrm{E}_{2}-20 \mathrm{~W}$ and $\mathrm{E}_{2}-25 \mathrm{~W}$ groups and the positive rates of the $\mathrm{E}_{2}$-injected rabbits were almost double those of the normal ones. On the other hand, the positive ratios in the resting and proliferating zones were much smaller. Significantly higher positive ratios compared to the normal rabbits were detected only in the resting zones of $\mathrm{E}_{2}-20 \mathrm{~W}$ and $\mathrm{E}_{2}-25 \mathrm{~W}$ rabbits and in the proliferating zone of $\mathrm{E}_{2}-15 \mathrm{~W}$ and $\mathrm{E}_{2}-25 \mathrm{~W}$ rabbits.

PCNA-positive chondrocytes with diffuselystained nuclei were found mainly in the proliferating and the upper hypertrophic zones of every growth plate as in our previous studies (Fig. 8) (Aizawa et al. 1997; Irie et al. 2005). The PCNApositive ratio of the whole growth plate tended to decrease with age in both the normal and ovariectomized rabbits, while the ratio of $\mathrm{E}_{2}$-injected rabbits was less than 2\% in each age group (Fig. 9). Statistically, the $\mathrm{E}_{2}$-injected rabbits showed the lowest rates, while the ovariectomized rabbits had lower positive rates than the normal ones in the whole growth plate and in the three divided zones.

\section{Discussion}

Ovariectomy with or without subsequent estrogen injection has been one of the major methodologies for investigating the effect of the female hormone, and several types of animals have been used for this method (Silberberg and Silberberg 1971; Weise et al. 2001; Nilsson et al. 2002, 2003). In rabbits, sexual maturation starts around four months after birth (Suzuki et al. 1989). The current study showed that the serum 


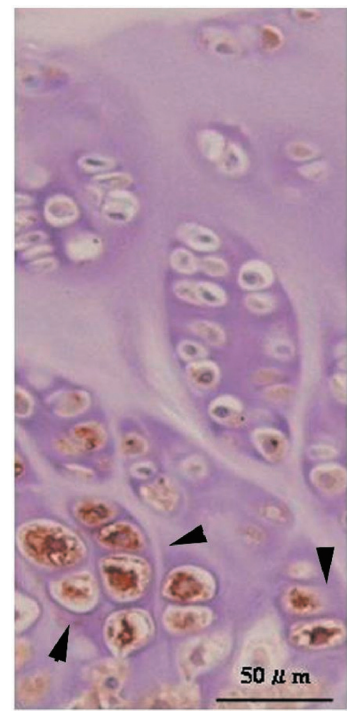

A

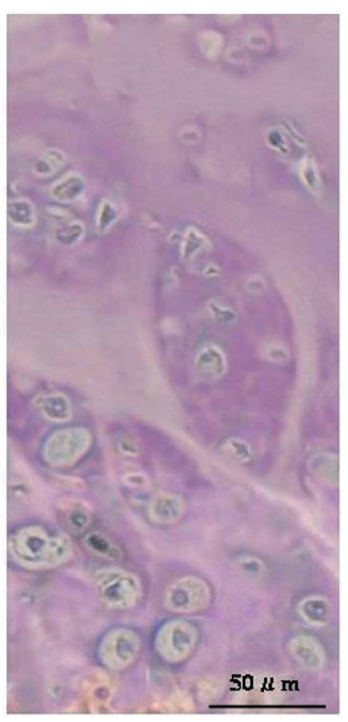

B

Fig. 6. Photomicrographs showing immunostaining for caspase- 3 in the growth plate of an ovariectomized 20-week-old rabbit and its negative control. A: The positive chondrocytes (arrowheads) are mainly detected in the hypertrophic zone. B: No positive cells are found in the negative control. Original magnification $\times 200$.
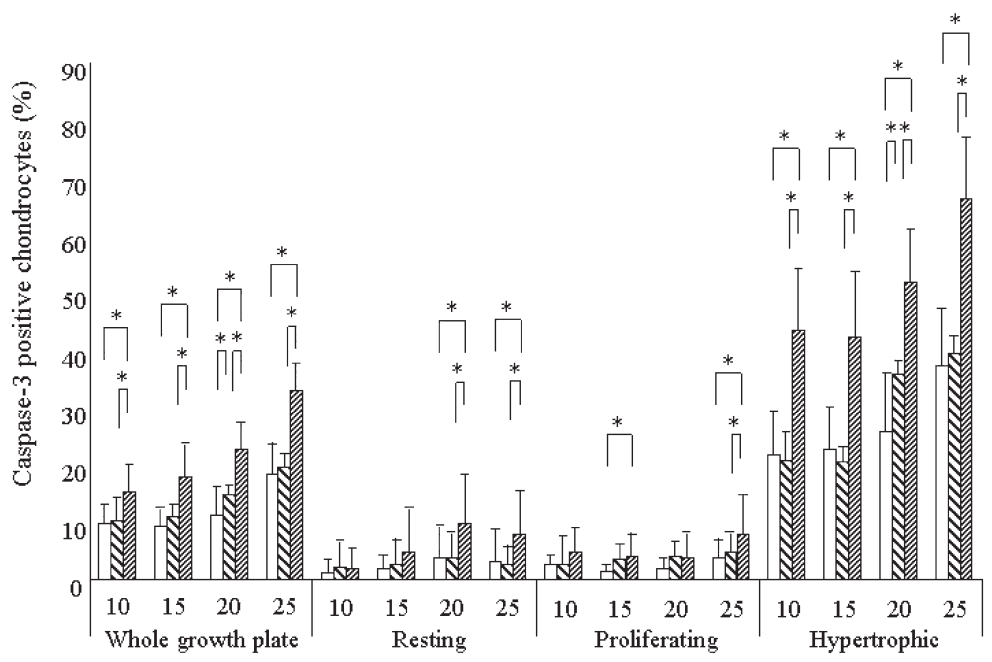

Fig. 7. Histogram showing the mean (s.D.) caspase-3-positive ratios in the whole growth plate and three divided zones in the normal, ovariectomized, and $\mathrm{E}_{2}$-injected rabbits. Each bar represents the mean (s.D.). The ratios of the whole growth plate tend to increase with age in all three rabbit groups. The $\mathrm{E}_{2}$-injected rabbits show the highest positive ratios in every age group, and they are significantly higher than those of the other two rabbit groups at the same age. Significant difference between each group is: $* p<0.05$.

$\square$, normal rabbits; $\mathbb{N}$, ovariectomized rabbits; 囷, $\mathrm{E}_{2}$-injected rabbits. 


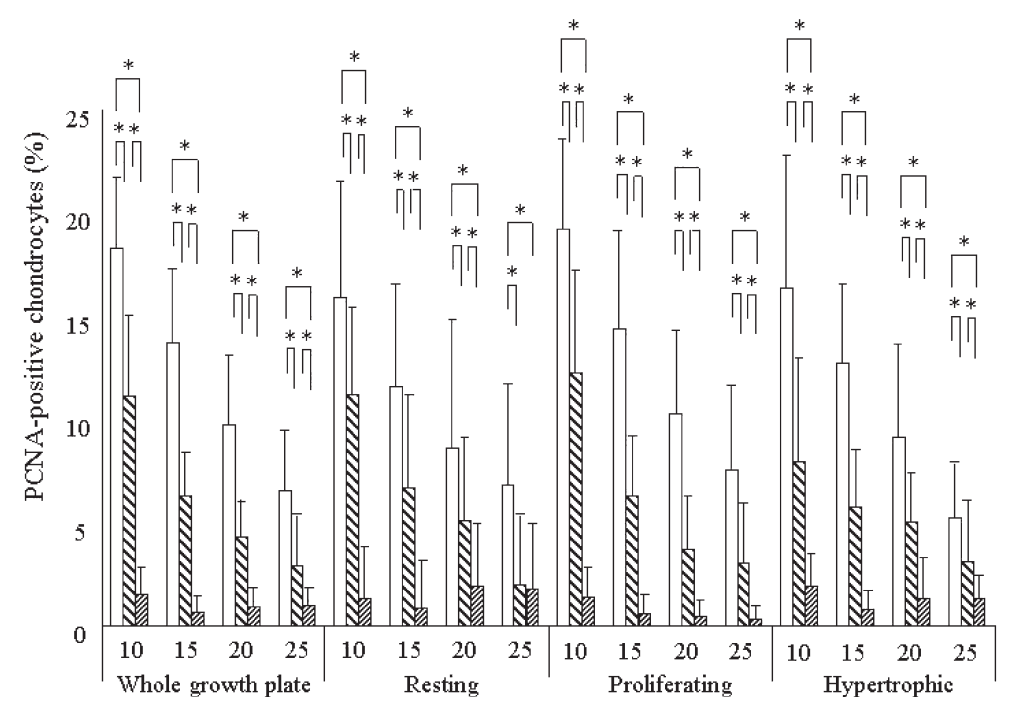

Fig. 9. Histogram showing the mean (s.D.) PCNA-positive ratios in the whole growth plate and three divided zones. The ratios decrease with age in the whole growth plate and three divided zones of the normal, ovariectomized, and $\mathrm{E}_{2}$-injected rabbits. Statistically, the $\mathrm{E}_{2}$-injected rabbits show the lowest rates, while the ovariectomized rabbits have lower positive rates than the normal ones in the whole growth plate and in the three divided zones. Significant difference between each group is: $* p<0.05$.

$\square$, normal rabbits; $\mathbb{N}$, ovariectomized rabbits; $\mathbb{Z}, \mathrm{E}_{2}$-injected rabbits.

$\mathrm{E}_{2}$ levels of ovariectomized rabbits were less than those of the normal ones before puberty. Thus, the growth plates of the former were not exposed to high levels of $E_{2}$. On the other hand, the $E_{2}$ levels of the all $\mathrm{E}_{2}$-injected rabbits were more than those of the normal ones at 25 weeks, indicating that those rabbits were exposed to supraphysiologic levels of $E_{2}$. Therefore, the timing of ovariectomy and also the dose of $\mathrm{E}_{2}$ injection used in the present study resulted in either very low or extremely high estrogen levels.

The overall growth plate height is mainly determined by the production of an extracellular matrix and the volume increase of hypertrophic cells (Robertson 1990; Farnum et al. 2002; van der Eerden et al. 2003; Roach et al. 2003). Hence a reduction in the number of hypertrophic chondrocytes should affect the growth plate height. Additionally, inhibition of extracellular matrix synthesis by high dose of estrogen would be expected to reduce the height in the E2-injected rabbits (Hunziker 1988; van der Eerden et al. 2003). However, in spite of the decline in chondrocyte numbers, the ovariectomized rabbits showed an increase in growth plate height. One explanation might be that matrix synthesis is more strongly affected than cell kinetics.

Previous studies found that ovariectomy accelerates, while subsequent high dose of estrogen administration suppresses, long bone growth (Silberberg and Silberberg 1971; Nilsson et al. 2002; Chagin et al. 2004; Nilsson and Baron 2005). This was confirmed in the present study in that the heights of the growth plates and overall lengths of the femurs were greatest in the ovariectomized group. Interestingly, however, both low and high estrogen levels led to reduced chondrocyte numbers, with the $\mathrm{E}_{2}$-injected rabbits showing a more dramatic effect. This suggested that different pathways are used depending on low or high dose of estrogen.

Estrogen can bind to two different receptors (Kousteni et al. 2001; Ito 2007), which exerts opposite effects on longitudinal bone growth. ER-alpha accelerates endochondral bone formation and contributes to the growth spurt in puberty, whereas ER-beta represses long bone growth (Lindberg et al. 2001; Chagin et al. 2004; Nilsson 
et al. 2005). A recent study indicated that ERalpha upregulated cyclin D1 and PCNA, which stimulated cell progression and prevented the apoptotic cascade, while ER-beta downregulated proliferation and had pro-apoptotic properties (Taylor et al. 2006). Which receptor, ER-alpha or ER-beta, dominates in the regulation of skeletal growth depends on the sex, species, and even the dose of estrogen (Kusec et al. 1998; van der Eerden et al. 2002; Chagin et al. 2004). In humans, the effects of estrogen are primarily mediated by ER-alpha, but higher doses during puberty lead to growth plate fusion, which may be mediated through ER-beta (Nilsson et al. 2005).

In addition, estrogen acts via non-ER pathways, including the growth hormone-insulin like growth factor-1 (GH-IGF-1) axis and protein kinase C (PKC) (van der Eerden et al. 2002; McMillan et al. 2006). Estrogen increases GH secretion by the pituitary, and GH and the subsequent increase in the local generation of IGF-1 stimulate cell proliferation in the growth plate (van der Eerden et al. 2002; Eastell 2005; Nilsson et al. 2005; Nilsson and Baron 2005). PKC activation can initiate a signaling cascade that causes changes in gene expression through multiple mitogenactivated protein kinase (MAPK) signaling pathways including ERK1/2 and p38 (Raucci et al. 2004; McMillan et al. 2006). ERK1/2 are usually implicated in cell proliferation, differentiation, and survival, whereas p38 might be required for chondrocyte hypertrophy and apoptosis (Raucci et al. 2004; McMillan et al. 2006). Likewise, rapid transient increases in MAPK are associated with proliferation, whereas persistent elevation in MAPK is associated with chondrocyte growth arrest and differentiation (Raucci et al. 2004; McMillan et al. 2006).

In this study, the excess $\mathrm{E}_{2}$ in the $\mathrm{E}_{2}$-injected rabbits might bind to ER-beta and/ or initiate p38 MAPK, which would be stronger than the action through the GH-IGF-1 axis. Thus, chondrocyte numbers decreased. On the other hand, normal level of estrogen might bind to ER-alpha in chondrocytes and/ or activate GH-IGF-1 axis and/ or ERK1/2 MAPK. Once estrogen is deficient, as in the ovariectomized rabbits, activation of these pathways might not be activated. Therefore, the ovariectomized rabbits also showed a decrease in the numbers of chondrocytes.

In spite of the decline in the number of chondrocytes, ovariectomy did not shorten the femur compared to the normal rabbits. Skeletal maturation as a consequence of both estrogen-induced vascular and osteoblastic invasion, and the termination of chondrogenesis might be delayed in the ovariectomized group, because ER-beta is closely related to this process (Grumbach 2000; Lindberg et al. 2001; van der Eerden et al. 2002; Chagin et al. 2004; Nilsson et al. 2005). In addition, extracellular matrix synthesis would be accelerated. Thus, the growth plate might not fuse and chondrogenesis and osteogenesis might continue slowly, which might lead to an increase in longitudinal bone growth although the exact mechanism of the contradictive results of ovariectomy to cell kinetics and long bone growth are still uncertain.

\section{Acknowledgements}

The authors wish to acknowledge the technical assistance of Katsuyoshi Shoji for his help in this study.

\section{References}

Aizawa, T., Kokubun, S. \& Tanaka, Y. (1997) Apoptosis and proliferation of growth plate chondrocytes in rabbits. $J$. Bone Joint Surg., 79-B, 483-486.

Aizawa, T., Roach, H.I., Kokubun, S. \& Tanaka, Y. (1998) Changes in the expression of Fas, osteonectin and osteocalcin with age in the rabbit growth plate. J. Bone Joint Surg., 80-B, 880-887.

Aizawa, T., Kokubun, S., Kawamata, T., Tanaka, Y. \& Roach, H.I. (1999) c-Myc protein in the rabbit growth plate. Changes in immunolocalisation with age and possible roles from proliferation to apoptosis. J. Bone Joint Surg., 81-B, 921-925.

Bardin, A., Hoffmann, P., Boulle, N., Katsaros, D., Vignon, F., Pujol, P. \& Lazennee, G. (2004) Involvement of estrogen receptor beta in ovarian carcinogenesis. Cancer Res., 64, 5861-5869.

Chagin, A.S., Lindberg, M.K., Andersson, N., Moverare, S., Gustafsson, J.A., Savendahl, L. \& Ohlsson, C. (2004) Estrogen receptor-beta inhibits skeletal growth and has the capacity to mediate growth plate fusion in female mice. $J$. Bone Miner. Res., 19, 72-77.

Chrysis, D., Nilsson, O., Ritzen, E.M. \& Savendahl, L. (2000) Apoptosis is developmentally regulated in rat growth plate. Endocrine., 18, 271-278.

Eastell, R. (2005) Role of oestrogen in the regulation of bone turnover at the menarche. J. Endocrinol., 185, 223-234.

Farnum, C.E., Lee, R., O'Hara, K. \& Urban, J.P. (2002) Volume increase in growth plate chondrocytes during 
hypertrophy: the contribution of organic osmolytes. Bone, 30, 574-581.

Grumbach, M.M. (2000) Estrogen, bone, growth and sex: a sea change in conventional wisdom. J. Pediatr. Endocrinol. Metab., 13, 1439-1455.

Hunziker, E.B., Schenk, R.K. \& Cruz-Orive, L.M. (1987) Quantitation of chondrocyte performance in growth-plate cartilage during longitudinal bone growth. J. Bone Joint Surg., 69-A, 162-173.

Hunziker, E.B. (1988) Growth plate structure and function. Pathol. Immunopathol. Res., 7, 9-13.

Irie, T., Aizawa, T. \& Kokubun, S. (2005) The role of sex hormones in the kinetics of chondrocytes in the growth plate. A study in the rabbit. J. Bone Joint Surg., 87-B, 1278-1284.

Ito, K. (2007) Hormone replacement therapy and cancers: the biological roles of estrogen and progestin in tumorigenesis are different between the endometrium and breast. Tohoku J. Exp. Med., 212, 1-12.

Korenman, S.G., Tulchinsky, D. \& Eaton, L.W., Jr. (1970) Radio-ligand procedures for estrogen assay in normal and pregnancy plasma. Acta Endocrinol. Suppl. (Copenh), 147, 291-304.

Kousteni, S., Bellido, T., Plotkin, L.I., O’Brien, C.A., Bodenner, D.L., Han, L., Han, K., DiGregorio, G.B., Katzenellenbogen, J.A., Katzenellenbogen, B.S., Roberson, P.K., Weinstein, R.S., Jilka, R.L. \& Manolagas, S.C. (2001) Nongenotropic, sex-nonspecific signaling through the estrogen or androgen receptors: dissociation from transcriptional activity. Cell, 104, 719-730.

Kusec, V., Virdi, A.S., Prince, R. \& Triffitt, J.T. (1998) Localization of estrogen receptor-alpha in human and rabbit skeletal tissues. J. Clin. Endocrinol. Metab., 83, 24212428.

Lindberg, M.K., Alatalo, S.L., Halleen, J.M., Mohan, S., Gustafsson, J.A. \& Ohlsson, C. (2001) Estrogen receptor specificity in the regulation of the skeleton in female mice. J. Endocrinol., 171, 229-236.

McMillan, J., Fatehi-Sedeh, S., Sylvia, V.L., Bingham, V., Zhong, M., Boyan, B.D. \& Schwartz, Z. (2006) Sexspecific regulation of growth plate chondrocytes by estrogen is via multiple MAP kinase signaling pathways. Biochim. Biophys. Acta, 1763, 381-392.

Nilsson, O., Abad, V., Chrysis, D., Ritzen, E.M., Savendahl, L. \& Baron, J. (2002) Estrogen receptor-alpha and -beta are expressed throughout postnatal development in the rat and rabbit growth plate. J. Endocrinol., 173, 407-414.

Nilsson, O., Falk, J., Ritzen, E.M., Baron, J. \& Savendahl, L. (2003) Raloxifene acts as an estrogen agonist on the rabbit growth plate. Endocrinol., 144, 1481-1485.

Nilsson, O. \& Baron, J. (2005) Impact of growth plate senescence on catch-up growth and epiphyseal fusion. Pediatr. Nephrol., 20, 319-322.
Nilsson, O., Marino, R., De Luca, F., Phillip, M. \& Baron, J. (2005) Endocrine regulation of the growth plate. Horm. Res., 64, 157-165.

Oestergaard, S., Sondergaard, B.C., Hoegh-Anderson, P., Henriksen, K., Qvist, P., Christiansen, C., Tankó, L.B. \& Karsdal, M.A. (2006) Effects of ovariectomy and estrogen therapy on type II collagen degradation and structural integrity of articular cartilage in rats. Arthritis Rheum., 54, 2441-2451.

Pacifici, M., Oshima, O., Fisher, L.W., Young, M.F., Shapiro, I.M. \& Leboy, P.S. (1990) Changes in osteonectin distribution and levels are associated with mineralization of the chicken tibial growth cartilage. Calcif. Tissue Int., 47, 51-61.

Roach, H.I., Mehta, G., Oreffo, R.O.C., Clarke, N.M.P. \& Cooper, C. (2003) Temporal analysis of rat growth plates: cessation of growth with age despite presence of a physis. $J$. Histochem. Cytochem., 51, 373-383.

Raucci, A., Laplantine, E., Mansukhani, A. \& Basilico, C. (2004) Activation of the ERK1/2 and p38 mitogen-activated protein kinase pathways mediates fibroblast growth factorinduced growth arrest of chondrocytes. J. Biol. Chem., 279, 1747-1756.

Robertson, W.W., Jr. (1990) Newest knowledge of the growth plate. Clin. Orthop., 253, 270-278.

Rodd, C., Jourdain, N. \& Alini, M. (2004) Action of estradiol on epiphyseal growth plate chondrocytes. Calcif. Tissue Int., 75, 214-224.

Silberberg, M. \& Silberberg, R. (1971) Steroid hormones and bone. In: The Biochemistry and Physiology of Bone, edited by Bourne, G.H., Vol. 3, 2nd ed., New York, Academic Press Inc., 401-484.

Suzuki, K., Kanno, S., Sudou, K., Takagaki, Y., Tanaka, M., Nakagawa, M., Oriya, T. \& Yamada, J. (1989) Rabbit. In: Basic Laboratory Animal Science and Technology, edited by Japanese Laboratory Animal Society, Vol. 2, Tokyo, Maruzen Company, 97-119. (in Japanese)

Taylor, R.A., Cowin, P., Couse, J.F., Korach, K.S. \& Risbridger, G.P. (2006) 17beta-estradiol induces apoptosis in the developing rodent prostate independently of ER-alpha or ER-beta. Endocrinol., 147, 191-200.

Van der Eerden, B.C.J., Gevers, E.F., Lowik, C.W.G.M., Karperien, M. \& Wit, J.M. (2002) Expression of estrogen receptor alpha and beta in the epiphyseal plate of the rat. Bone, 30, 478-485.

Van der Eerden B.C.J., Karperien, M. \& Wit, J.M. (2003) Systemic and local regulation of the growth plate. Endocr. Rev., 24, 782-801.

Weise, M., De-Levi, S., Barnes, K.M., Gafni, R.I., Abad, V. \& Baron, J. (2001) Effects of estrogen on growth plate senescence and epiphyseal fusion. Proc. Natl. Acad. Sci. USA, 98, 6871-6876. 